\title{
Survival after bidirectional cavopulmonary anastomosis: Analysis of preoperative risk factors
}

\author{
Mark A. Scheurer, MD, ${ }^{a}$ Elizabeth G. Hill, PhD, ${ }^{d}$ Nagavardhan Vasuki, MD, ${ }^{\mathrm{b}}$ Scott Maurer, MD, ${ }^{\mathrm{c}}$ Eric M. Graham, MD, \\ Varsha Bandisode, MD, ${ }^{e}$ Girish S. Shirali, MBBS, ${ }^{e}$ Andrew M. Atz, MD, ${ }^{e}$ and Scott M. Bradley, MD ${ }^{f}$
}

Supplemental material is available online.
From the Department of Cardiology, Children's Hospital Boston, Boston, Mass ; ; Department of Medicine, Emory University, Atlanta, $\mathrm{Ga}^{\mathrm{b}}$; Department of Pediatrics, Naval Medical Center, San Diego, Calif ${ }^{c}$; and Department of Biostatistics, Bioinformatics \& Epidemiology, ${ }^{\mathrm{d}}$ Department of Pediatrics, Division of Cardiology, ${ }^{e}$ and Department of Surgery, Division of Cardiothoracic Surgery, ${ }^{\mathrm{f}}$ Medical University of South Carolina, Charleston, SC.

Received for publication Dec 21, 2006; revisions received Jan 12, 2007; accepted for publication Feb 5, 2007.

Address for reprints: Mark A. Scheurer, MD, Instructor of Pediatrics, Harvard Medical School, Cardiac Intensivist, Department of Cardiology, Children's Hospital Boston, Bader 660, 300 Longwood Ave, Boston, MA 02115 (E-mail: mark.scheurer@cardio. chboston.org).

J Thorac Cardiovasc Surg 2007;134:82-9

$0022-5223 / \$ 32.00$

Copyright (๑) 2007 by The American Association for Thoracic Surgery

doi:10.1016/j.jtcvs.2007.02.017
Objective: Prognostic factors for survival after bidirectional cavopulmonary anastomosis for functionally single ventricle are not well defined. We analyzed preoperative hemodynamic and echocardiographic data to determine risk factors for death or transplantation at least 1 year after bidirectional cavopulmonary anastomosis.

Methods: Data for all patients who underwent bidirectional cavopulmonary anastomosis before 5 years of age at our institution from September 1995 through June 2005 were analyzed. Available preoperative echocardiograms and catheterizations were reviewed. Survivors were compared with those who died or underwent transplantation. Bivariable associations between demographic and clinical risk factors and survival status (alive without transplantation vs dead or transplanted) were assessed with Wilcoxon rank sum test and $\chi^{2}$ or Fisher exact tests. Survival functions were constructed with Kaplan-Meier estimates, and event times compared between subgroups with log-rank tests. Cox proportional hazard modeling was used for multivariable modeling of risk of death or transplantation.

Results: One hundred sixty-seven patients underwent bidirectional cavopulmonary anastomosis with hemi-Fontan $(\mathrm{n}=62)$ or bidirectional Glenn $(\mathrm{n}=105)$ operations. Three patients died before discharge, 11 died later, and 1 has undergone transplantation. Freedom from death or transplantation after bidirectional cavopulmonary anastomosis was $96 \%$ at 1 year and $89 \%$ at 5 years. Multivariable analysis of preoperative variables showed atrioventricular valve regurgitation to be an independent risk factor for death or transplantation (hazard ratio 2.8, 95\% confidence interval 1.1-7.1, $P=.02$ ).

Conclusion: Although survival after bidirectional cavopulmonary anastomosis is high, preoperative atrioventricular valve regurgitation is an important risk factor for death or transplantation.

$\mathrm{T}$ he creation of a bidirectional cavopulmonary anastomosis (BCPA) for the patient with a functionally single ventricle is an effective strategy for intermediate staged palliation before the Fontan procedure. ${ }^{1}$ Initially designed as the final surgical procedure for patients with single-ventricle physiology, the BCPA evolved into an intermediate staging procedure for those patients who were deemed at high risk for proceeding directly to the Fontan procedure. ${ }^{2,3}$ With mounting evidence of improvements in survival and morbidity with the staged approach, the BCPA is now routinely used by most centers as a staging procedure before Fontan completion. ${ }^{4}$

Although echocardiograms and elective hemodynamic catheterizations are routinely performed before BCPA in many cases, limited data exist in the literature defining prognostic factors for survival after BCPA. The goal of this study was to 


\section{Abbreviations and Acronyms}

$\mathrm{BCPA}=$ bidirectional cavopulmonary anastomosis

HLHS $=$ hypoplastic left heart syndrome

define which of the routinely collected preoperative variables were predictive of death or transplantation after BCPA at our institution.

\section{Materials and Methods \\ Patient Population}

The institutional review board for human research of the Medical University of South Carolina approved the use of patient medical records for this retrospective review. From 1995 through the present, our institution has routinely performed BCPA in patients with singleventricle physiology as staged palliation before the Fontan procedure. From September 1, 1995, through June 30, 2005, a total of 167 patients 5 years old or younger with single-ventricle physiology underwent BCPA performed by a single surgeon (S.M.B.) at the Medical University of South Carolina. At the discretion of the surgeon, these patients underwent bidirectional Glenn or hemi-Fontan operations. Data from all 167 patients are included in the analysis. Patient demographic characteristics, cardiac anatomic morphology, perioperative variables, and hospital course were reviewed for each patient. All patients' courses were followed until death, transplantation, unavailability for follow-up, or June 30, 2006, whichever came first. A patient's time to death or transplantation was censored if the patient was lost to follow-up or did not experience transplantation or die by the end of the follow-up period.

\section{Study Design}

Complete two-dimensional, pulse-wave Doppler, and color Doppler echocardiograms and complete hemodynamic catheterizations are routinely performed before BCPA at our institution. All available echocardiographic and catheterization reports before BCPA were retrospectively reviewed for each patient. One hundred seventeen echocardiograms (including all performed after March 1999) and 163 catheterizations were sufficiently complete to allow for data analysis.

\section{Echocardiograms}

The last complete echocardiogram before BCPA for each patient was included for analysis. Subjective grades for both atrioventricular valve regurgitation and systemic semilunar regurgitation were reported on an ordinal scale from 0 (none) to 4 (severe) in half-unit increments. Similarly, ventricular function of the systemic ventricle was graded on an ordinal scale from 0 (severe dysfunction) to 4 (normal) in half-unit increments.

\section{Catheterizations}

All hemodynamic data were gathered retrospectively from reports produced at the time of the catheterization. Concomitant procedures, including balloon or blade atrial septostomy and balloon dilation of coarctation, were recorded. For those with interventional procedures, hemodynamic data recorded after the procedure were included in the analysis.

\section{Statistical Analysis}

Measures of central tendency and dispersion are expressed in terms of median and range (rather than mean and SD) because of observed skewness in the distributions of predictor variables. Bivariable associations between demographic or clinical risk factors and survival status (alive versus dead or having undergone transplantation) were assessed with Wilcoxon rank sum test and $\chi^{2}$ or Fisher exact tests for continuous and categorical variables, respectively. Survival functions were constructed with Kaplan-Meier estimates, and event times were compared between subgroups with log-rank tests.

To accommodate multivariable modeling, a multiple imputation model with predictive mean matching was used to augment missing covariate data. ${ }^{5}$ Multiple imputation has been shown to produce efficient and unbiased estimates if the data are missing at random. ${ }^{5,6}$ Specifically, we assume that the quality of being missing was possibly associated with other observed variables but was not related to the value of the partially observed variable itself. In our data, missing covariate information was primarily attributable to the year in which the patient underwent BCPA but was unrelated to the variable's value, thus satisfying the missing at random assumption. According to recommendations that the imputation model contain a comprehensive subset of variables, we constructed the multiply imputed data from a model containing the following: all perioperative variables (with the exception of balloon dilation of coarctation and atrial septostomy), a variable indicating whether the patient underwent BCPA before 2000, the time to death or transplantation, and a censoring indicator. ${ }^{7}$ Subsequent regressions that were based on the imputation data with Cox proportional hazard modeling were used to identify independent risk factors for death or transplantation. Risk estimates were obtained as the average across the regressions, with corresponding interval estimates reflecting both within- and between-imputation variability.

The number of hazard regression model covariates was limited by the total number of events (deaths or transplants) in the study. ${ }^{8,9}$ Because of the small number of events in this analysis (15 total), we considered for inclusion in the model only those perioperative variables found to be significant in univariate analyses. The presence of collinearity between predictors was investigated; if present, one variable was excluded from the final model. Both the proportional hazards assumption and the functional form of continuous model variables were assessed according to cumulative sums of martingale-based residuals. ${ }^{10}$

All statistical tests were 2-sided, and type I error was controlled at a level of .05. Analyses were performed with SAS (version 9.1; SAS Institute, Inc, Cary, NC), SPSS (version 14.0; SPSS Inc, Chicago, Ill), and Sigmaplot (version 10.0; Systat Software, Inc, Point Richmond, Calif). Multiply imputed data sets were generated with the MICE package ${ }^{11}$ in R (R Development Core Team, 2005).

\section{Results \\ Patient Outcomes}

Of the 167 patients who underwent BCPA, 3 died within 30 days of the operation (Figure 1). One of these patients had Ebstein anomaly with pronounced atrialization of the right ventricle and severe tricuspid regurgitation and was unable to be weaned from extracorporeal membrane oxygenation after a bidirectional Glenn operation, modified Blalock- 


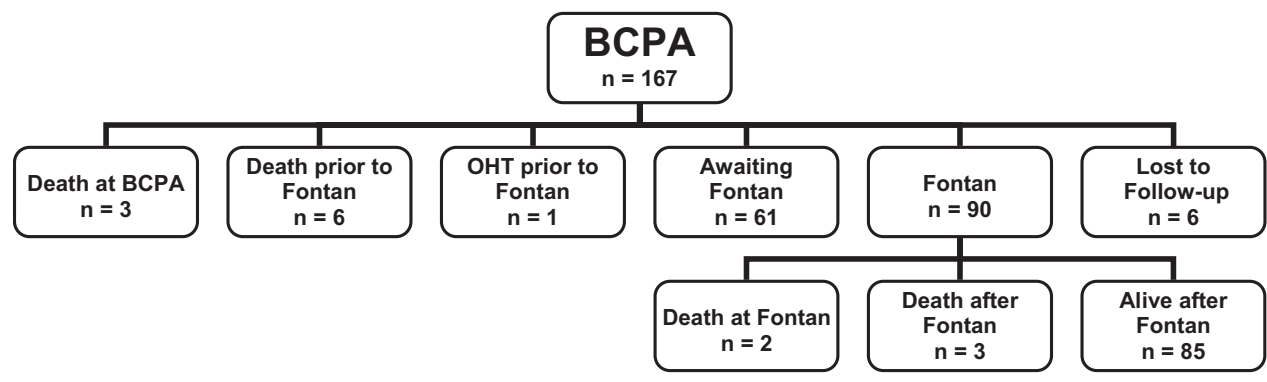

Figure 1. Outcomes of 167 patients who underwent bidirectional cavopulmonary anastomosis (BCPA). Minimum follow-up for all patients was 1 year or occurrence of death or transplantation (OHT).

Taussig shunt, and tricuspid valvuloplasty. The other 2 patients had hypoplastic left heart syndrome (HLHS): 1 with bilateral superior venae cavae and moderate to severe tricuspid regurgitation requiring a tricuspid valvuloplasty and profound refractory cyanosis and the other with postoperative mediastinitis and peritonitis.

Six other patients died before undergoing a Fontan procedure, at a median of 3.9 months (1.3-46 months) after BCPA. One with HLHS, moderate tricuspid regurgitation, and poor ventricular function died 1.5 months after BCPA, tricuspid valvuloplasty, and Blalock-Taussig shunt. Another patient with HLHS and severe tricuspid regurgitation died 3.3 months after BCPA and tricuspid valvuloplasty while being considered for transplantation. A patient with double-inlet left ventricle morphology died for unknown reasons while at home 1.3 months after an uncomplicated BCPA. A patient with an unbalanced atrioventricular septal defect and moderate common atrioventricular valve regurgitation died 4.3 months after BCPA and common atrioventricular valvuloplasty. A 4-year-old with a double-inlet left ventricle, severe ventricular dysfunction, and aortic regurgitation died during catheterization to evaluate for transplantation candidacy 3 years after BCPA. More than 3.5 years after BCPA, a 5-year-old child with trisomy 21, an unbalanced atrioventricular septal defect, and bilateral superior venae cavae died. This child had undergone a common atrioventricular valvuloplasty and Blalock-Taussig shunt insertion 1 year after BCPA.

At 5 years of age, nearly 4.5 years after BCPA, 1 patient with HLHS, severe ventricular dysfunction, and tricuspid regurgitation underwent orthotopic heart transplantation. This patient is alive at a follow-up of 18 months. Six patients became unavailable for follow-up before a planned Fontan operation $(3.6 \%)$.

Ninety patients have undergone a fenestrated Fontan procedure at a median of 3.3 years of age (1.1-7.7 years). Eighty-five of these patients are alive at a median follow-up of 3.8 years (4.7 months-9.2 years) from the time of Fontan completion. Two patients died within 1 month of the Fontan procedure, and 3 died remotely at 2 months and at 1.4 and 4.4 years from the time of Fontan completion. In the perioperative period, 1 child died as the result of mediastinitis and another child, with severe developmental delay, died as the result of aspiration events. Of the 3 remote deaths, 1 was as a result of a hypercoagulable state with resultant systemic thromboembolic events across the fenestration, 1 was as a result of profound protein-losing enteropathy during consideration for transplantation, and 1, of a child with trisomy 21, was caused by severe ventricular dysfunction, elevated Fontan pressures, and esophageal varices culminating in profound esophageal variceal bleeding.

The overall freedoms from death or transplantation for all patients were $96 \%$ at one year and $89 \%$ at 5 years (Figure 2). The median follow-up for all patients was 4.4 years ( 3 days10.7 years).

Freedom from Death or Transplantation

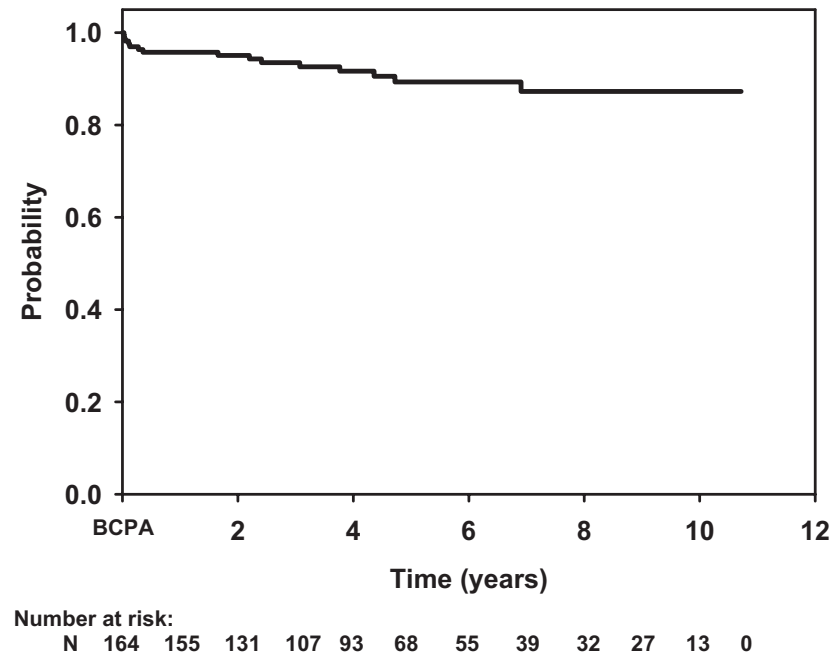

Figure 2. Kaplan-Meier plot of freedom from death or transplantation after bidirectional cavopulmonary anastomosis (BCPA) for entire cohort $(n=167)$. 


\section{Demographic and Morphologic Characteristics}

Bivariable associations of demographic variables with survival status revealed no statistically significant differences between those who lived and those who died or underwent transplantation (Table 1). Among anatomic subtypes, only those who had the diagnosis of an unbalanced atrioventricular septal defect or the presence of bilateral superior venae cavae were more likely to have died or undergone transplantation. Among the study patients with bilateral superior venae cavae, 2 patients underwent ligation of a small accessory vena cava at the time of hemi-Fontan, whereas all others had bilateral BCPA performed.

No patient with tricuspid atresia $(n=27)$ or pulmonary atresia and intact ventricular septum $(\mathrm{n}=4)$ who underwent BCPA died or received a transplant in follow-up.

\section{Preoperative Echocardiography and Catheterization}

Analyses of preoperative echocardiograms revealed that those who remained alive had less atrioventricular valve regurgitation than did those who died or underwent transplantation (Table 2). Those remaining alive after BCPA had lower preoperative end-diastolic pressure of the systemic ventricle, lower transpulmonary gradient, and lower pulmonary vascular resistance than did those who died or underwent transplantation.

\section{Perioperative Variables}

Proportionally, more patients who died or underwent transplantation (7/15) than those remaining alive (12/ 152) underwent repair of the systemic atrioventricular valve during BCPA $(P<.001)$ (Table 3$)$. Seventeen of the 117 patients in this study with adequate preoperative echocardiograms for analysis were found to have at least moderate atrioventricular valve regurgitation. Thirteen of these $17(76 \%)$ underwent repair of the valve at the time of BCPA. Among those 13 patients who underwent repair of the atrioventricular valve and had at least moderate atrioventricular valve regurgitation, 10 had HLHS, 3 had an unbalanced atrioventricular septal defect, and 1 had Ebstein anomaly; 2 with HLHS and 2 with an unbalanced atrioventricular septal defect died. Among the 4 patients who had at least moderate atrioventricular valve regurgitation by echocardiography but did not undergo an atrioventricular valvuloplasty at the time of BCPA, 1 has undergone orthotopic heart transplantation.

Three of the 15 patients who have died or undergone transplantation had an additional source of pulmonary blood flow through the insertion of a systemic-pulmonary shunt or maintenance of native antegrade pulmonary blood flow, as opposed to only 2 of the event-free survivors $(P<.01)$. Of the 4 patients who had a modified Blalock-Taussig shunt placed at the time of BCPA, only 1 was alive at follow-up. Before the operation, all 4 of these patients had relatively
TABLE 1. Demographic and cardiac morphologic characteristics of patients undergoing bidirectional cavopulmonary anastomosis

\begin{tabular}{|c|c|c|c|}
\hline Characteristic & $\begin{array}{c}\text { Alive } \\
(n=152)\end{array}$ & $\begin{array}{c}\text { Death or OHT } \\
(n=15)\end{array}$ & $P$ value \\
\hline \multicolumn{4}{|l|}{ Demographic } \\
\hline Age at anastomosis (mo) & $6.7(2.4-31.8)$ & $8.0(3.0-15.9)$ & .43 \\
\hline $\begin{array}{l}\text { Weight at catheterization } \\
(\mathrm{kg})\end{array}$ & $6.1(3.4-12.1)^{*}$ & $5.9(4.1-10.7)$ & .47 \\
\hline Male sex & $93(61 \%)$ & $10(67 \%)$ & .68 \\
\hline Era (2000-2005) & $97(64 \%)$ & $7(47 \%)$ & .19 \\
\hline \multicolumn{4}{|l|}{ Morphologic } \\
\hline Left ventricular dominance & $58(38 \%)$ & $4(27 \%)$ & .38 \\
\hline $\begin{array}{l}\text { Hypoplastic left heart } \\
\text { syndrome }\end{array}$ & $71(47 \%)$ & $8(53 \%)$ & .62 \\
\hline $\begin{array}{l}\text { Unbalanced } \\
\text { atrioventricular septal } \\
\text { defect }\end{array}$ & $10(7 \%)$ & $4(27 \%)$ & $.02 \dagger$ \\
\hline $\begin{array}{l}\text { Bilateral superior venae } \\
\text { cavae }\end{array}$ & $18(12 \%)$ & $5(33 \%)$ & $.04 \dagger$ \\
\hline $\begin{array}{l}\text { Interrupted inferior vena } \\
\text { cava }\end{array}$ & $8(5 \%)$ & $0(0 \%)$ & $>.999 \dagger$ \\
\hline
\end{tabular}

Values are median with range or number of patients for continuous and categorical variables, respectively. $P$ values are obtained from Wilcoxon rank-sum test (continuous variables) and $\chi^{2}$ test (categorical variables) except as noted. OHT, Orthotopic heart transplantation. ${ }^{*}$ Data for 150 patients. †Fisher exact test.

small pulmonary arteries according to the Nakata index and also had elevated mean pulmonary arterial pressures. Those who died or subsequently underwent transplantation were mechanically ventilated longer and had a longer total stay at the time of BCPA.

\section{Freedom From Death or Transplantation}

The Kaplan-Meier survival curves presented in Figures E1 through E6 show estimates of freedom from death or transplantation to 11 years after BCPA in patient groups dichotomized by certain preoperative variables. In each of these analyses, it appears that the probability of freedom from death or transplantation diverges for each set of patients at 2 to 3 years after BCPA. In this study, the median time to Fontan operation after BCPA was 2.5 years (0.5-6.7 years). To further evaluate the early risk of death or transplantation, separate survival analyses were done for all patients up to 3 years from BCPA. All dichotomized patient groups shown in Figures E1-E6 were also found to have significantly different transplantation-free survival rates up to 3 years after BCPA.

\section{Multivariable Analysis}

Table 4 summarizes results of the Cox proportional hazard modeling for 15 multiply imputed data sets. Multivariable analysis of preoperative variables showed only atrioventric- 
TABLE 2. Echocardiographic and catheterization variables before bidirectional cavopulmonary anastomosis

\begin{tabular}{|c|c|c|c|c|c|}
\hline \multirow[b]{2}{*}{ Variable } & \multicolumn{2}{|r|}{ Alive } & \multicolumn{2}{|c|}{ Death or transplantation } & \multirow[b]{2}{*}{$P$ value } \\
\hline & No. & Value & No. & Value & \\
\hline \multicolumn{6}{|l|}{ Echocardiographic } \\
\hline Atrioventricular valve regurgitation (0-4 ordinal scale) & 109 & & 8 & & \\
\hline Median & & $2(0-4)$ & & $3(1-3.5)$ & .01 \\
\hline Moderate or more $(\geq 3$, No.) & & $12(11 \%)$ & & $5(63 \%)$ & $<.01$ \\
\hline Semilunar valve regurgitation (0-4 ordinal scale) & 109 & & 8 & & \\
\hline Median & & $0(0-2.5)$ & & $0.5(0-2)$ & .10 \\
\hline Present $(>0$, No. $)$ & & $26(24 \%)$ & & $4(50 \%)$ & .20 \\
\hline Single ventricle function (0-4 ordinal scale) & 109 & & 8 & & \\
\hline Median & & $4(2-4)$ & & $4(4-4)$ & .31 \\
\hline \multicolumn{6}{|l|}{ Catheterization } \\
\hline Single-ventricle end-diastolic pressure $(\mathrm{mm} \mathrm{Hg})$ & 144 & & 15 & & \\
\hline Median & & $8(2-22)$ & & $11(7-17)$ & .02 \\
\hline$\geq 12 \mathrm{~mm} \mathrm{Hg}$ (No.) & & $25(17 \%)$ & & $7(47 \%)$ & .01 \\
\hline Transpulmonary gradient $(\mathrm{mm} \mathrm{Hg})$ & 132 & & 12 & & \\
\hline Median & & $6(1-36)$ & & $8.5(4-12)$ & .03 \\
\hline$\geq 9 \mathrm{~mm} \mathrm{Hg}$ (No.) & & $20(15 \%)$ & & $6(50 \%)$ & $<.01$ \\
\hline Pulmonary vascular resistance $\left(\mathrm{U} \cdot \mathrm{m}^{2}\right)$ & 135 & & 10 & & \\
\hline Median & & $1.8(0.3-7.1)$ & & $2.4(1.3-4.8)$ & .01 \\
\hline$\geq 2 \mathrm{U} \cdot \mathrm{m}^{2}$ (No.) & & $55(41 \%)$ & & $9(90 \%)$ & $<.01$ \\
\hline Mean pulmonary arterial pressure $(\mathrm{mm} \mathrm{Hg})$ & 134 & & 12 & & \\
\hline Median & & $13.3(6-42)$ & & $16.5(7-20)$ & .12 \\
\hline$\geq 18 \mathrm{~mm} \mathrm{Hg}$ (No.) & & $17(13 \%)$ & & $4(33 \%)$ & .07 \\
\hline Atrial pressure $(\mathrm{mm} \mathrm{Hg})$ & 139 & & 13 & & \\
\hline Median & & $7(1-15)$ & & $8(3-9)$ & .41 \\
\hline$\geq 7$ mm Hg (No.) & & $72(52 \%)$ & & $9(69 \%)$ & .26 \\
\hline Nakata index $\left(\mathrm{mm}^{2} / \mathrm{m}^{2}\right.$, median $)$ & 134 & $198.5(48-802)$ & 14 & $184.5(60-479)$ & .27 \\
\hline Ratio of pulmonary to systemic resistance (median) & 128 & $0.11(0.01-0.50)$ & 10 & $0.13(0.05-0.27)$ & .18 \\
\hline Ratio of pulmonary to systemic flow (median) & 137 & $1.16(0.23-4.70)$ & 11 & $1.04(0.37-1.69)$ & .24 \\
\hline Balloon dilatation of coarctation & 150 & $20(13 \%)$ & 15 & $2(13 \%)$ & $>.999$ \\
\hline Atrial septostomy & 150 & $6(4 \%)$ & 15 & $0(0 \%)$ & $>.999$ \\
\hline
\end{tabular}

$P$ values obtained with Wilcoxon rank-sum test (continuous variables) and Fisher exact test (categorical variables).

ular valve regurgitation to be an independent risk factor for death or transplantation (hazard ratio $2.8,95 \%$ confidence interval 1.1-7.1, $P=.02$ ). We considered the following for inclusion in the model: atrioventricular valve regurgitation, single-ventricle end-diastolic pressure, transpulmonary gradient, and pulmonary vascular resistance. There was evidence of moderate collinearity between pulmonary vascular resistance and transpulmonary gradient (Spearman correlation 0.6). With the additional concern of model parsimony, we excluded pulmonary vascular resistance from the model. Even with the imputed data, the total number of deaths or transplantations was small (15 events total), resulting in a rate of 5 events per variable in the final 3-variable model. This low event rate precluded investigation of variable interactions. Assessment of the proportional hazards assumption and the functional forms of the three model covariates according to cumulative sums of martingale residuals ${ }^{10}$ indicated adequate model fit.

\section{Discussion}

This study helps to define commonly collected preoperative patient characteristics that distinguish patients at highest risk for death or transplantation after BCPA. Despite the ventricular volume unloading associated with the BCPA and its possible favorable impact on atrioventricular annular dilation, preoperative atrioventricular valve regurgitation was found to be a significant risk factor for death or transplantation after BCPA. Although an increase in atrioventricular valve regurgitation has not been shown in other analyses $^{12,14}$ to be associated with less favorable outcomes, our study suggests it to be an important short- and long-term prognosticator. Although some patients with at least moderate atrioventricular valve regurgitation have fared well in our series and probably benefited from the hemodynamic consequences of BCPA, an important subgroup fared poorly.

Previous studies found no decrease in survival among patients who underwent repair of the systemic atrioventric- 
TABLE 3. Operative factors and postoperative course at bidirectional cavopulmonary anastomosis

\begin{tabular}{lccc}
\hline & $\begin{array}{c}\text { Alive } \\
(\mathbf{n}=\mathbf{1 5 2})\end{array}$ & $\begin{array}{c}\text { Death or 0HT } \\
(\mathbf{n}=\mathbf{1 5})\end{array}$ & $\boldsymbol{P}$ value \\
\hline $\begin{array}{c}\text { Operative factors } \\
\quad \text { Atrioventricular } \\
\quad \text { valvuloplasty (No.) }\end{array}$ & $\begin{array}{c}\text { Value } \\
\quad(8 \%)\end{array}$ & $\begin{array}{c}\text { Value } \\
7(47 \%)\end{array}$ & $<.001$ \\
$\begin{array}{c}\text { Additional pulmonary } \\
\quad \text { blood flow source (No.) }\end{array}$ & $2(1 \%)$ & $3(20 \%)$ & $<.01$ \\
$\quad$ Hemi-Fontan (No.) & $59(39 \%)$ & $5(33 \%)$ & .79 \\
$\begin{array}{c}\text { Postoperative course } \\
\text { Intubation time (d, median) }\end{array}$ & $1(0-54)$ & $3(1-39)$ & $<.001$ \\
$\quad$ Stay (d, median) & $8(4-103)$ & $14(5-166)$ & $<.01$ \\
\hline
\end{tabular}

$P$ values obtained from Wilcoxon rank sum test (continuous variables) and Fisher exact test (categorical variables). $\mathrm{OHT}$, Orthotopic heart transplantation.

ular valve at the time of BCPA. ${ }^{12,14}$ The need for atrioventricular valve repair of any type was collinear with atrioventricular valve regurgitation in our analysis and strongly associated with subsequent death or transplantation. This finding is consistent with the analysis of the multicenter Congenital Heart Surgeons Society database report of tricuspid valvuloplasty at BCPA in HLHS as an independent risk factor for death after BCPA. ${ }^{15}$

It is probable that both the exact anatomic mechanism of atrioventricular valve regurgitation (whether annular dilation, valve prolapse, chordal tethering, or another mechanism) and the specific surgical approaches to the regurgitation significantly affect survival after BCPA. In our study, because of the relatively small numbers of patients with heterogeneous anatomic diagnoses and unspecified mechanisms of valvular regurgitation, further meaningful analysis and interpretation of data from those who underwent valvuloplasty was not possible. In the future, prospective studies that delineate the exact mechanisms of atrioventricular valve regurgitation by means of emerging technologies, such as 3-dimensional echocardiography, and the prospective collection of the surgical methods used to correct the regurgitation will be essential in defining which subgroups of patients have more favorable outcomes.

The interactions of pulmonary arterial pressure, atrial pressure, atrioventricular valve regurgitation, and ventricular function contribute to the postoperative physiologic state in patients who undergo BCPA. In previous studies, elevated pulmonary arterial pressure after Glenn and Fontan procedures has been shown to be an independent marker of poor long-term outcome. ${ }^{12,16}$ In this study, neither preoperative atrial pressure, mean pulmonary arterial pressure, nor pulmonary vascular resistance was found to be independently associated with death or transplantation. We also examined transpulmonary gradient, which, representing the difference between pulmonary arterial and atrial pressure, incorporates both measurements. Although strongly associ-
TABLE 4. Multivariable analysis based on 15 data sets with missing data multiply imputed: Independent risk factors for death or transplantation after bidirectional cavopulmonary anastomosis $(n=167)$

\begin{tabular}{lccc}
\hline Preoperative variables & $\begin{array}{c}\text { Hazard } \\
\text { ratio* }\end{array}$ & $\begin{array}{c}\text { 95\% Confidence } \\
\text { interval }\end{array}$ & $\boldsymbol{P}$ value \\
\hline $\begin{array}{l}\text { Atrioventricular valve } \\
\quad \text { regurgitation }\end{array}$ & 2.8 & $1.1-7.1$ & .02 \\
$\begin{array}{l}\text { Transpulmonary gradient }(\mathrm{mm} \mathrm{Hg}) \\
\quad 1.1\end{array}$ & $1.0-1.2$ & .24 \\
$\quad \begin{array}{l}\text { Single-ventricle end-diastolic } \\
\quad \text { pressure }(\mathrm{mm} \mathrm{Hg})\end{array}$ & 1.1 & $0.9-1.2$ & .34 \\
\end{tabular}

Total number of deaths and transplantations was $15 .{ }^{*}$ Hazard ratios are based on a 1-unit increase in the value of the corresponding covariate.

ated with time to death or transplantation in univariate testing, transpulmonary gradient was not found to be independently associated with a poor outcome in this cohort.

Systemic ventricle end-diastolic pressure of at least $12 \mathrm{~mm}$ $\mathrm{Hg}$ was found to be associated with decreased survival in univariate analysis but was not found to be associated with poor outcome in multivariable testing. Because echocardiographically assessed ventricular function was not different between groups, investigations of the important relationships between ventricular function and end-diastolic pressure and atrioventricular valve regurgitation could not be made.

Previous studies of patients with complete cavopulmonary connections have shown heterotaxy syndrome to be associated with poor long-term survival. ${ }^{13}$ In our study, common anatomic components of either right or left atrial isomerism pertinent to the surgical approach required at BCPA were included for analysis. Those who underwent a Kawashima variation of BCPA because of an interrupted inferior vena cava with azygous continuation to a superior vena cava were at no increased risk of death or transplantation in follow-up when compared in univariate analysis with those who had an intact inferior vena cava. In univariate analysis, the presence of bilateral superior venae cavae (and thus either ligation of a superior vena cava or bilateral BCPAs at the time of second-stage palliation) and unbalanced atrioventricular septal defect were associated with death or transplantation. Multivariable analysis, however, did not elucidate an independent effect of either anatomic variant. In this study, no complete data existed to evaluate the influences of known chromosomal abnormalities, significant developmental delays, or the presence of pulmonary venous obstruction on transplantation-free survival after BCPA.

As suggested in separate actuarial transplantation-free survival analyses at 3 years of follow-up, at least some of the risks that each subgroup carries are borne before or at the typical timing of Fontan completion. The beneficial hemodynamic effects of BCPA for single-ventricle physiology have been well documented. ${ }^{17-19}$ Survival of patients 
ultimately destined to undergo the Fontan operation has been improved with the interposition of a BCPA operation. ${ }^{1}$ As defined by this and previous studies, some subgroups of patients after BCPA have decreased survival at short- and long-term follow-up. Orthotopic transplantation after a bidirectional Glenn or Fontan operation has been associated with 10-year survivals comparable to those of patients undergoing transplantation for other reasons. ${ }^{20}$ Further, Michielon and colleagues ${ }^{21}$ have suggested that BCPA could be considered the best bridge to transplantation for patients with failing single-ventricle physiology. With the identification and careful follow-up of patients at high risk for death, orthotopic transplantation could be carefully considered for selected patients before attempts at Fontan completion. The use of the BCPA as a final palliative surgical procedure, as suggested by some authors, was not evaluated in this study, because all patients were intended to receive Fontan completion provided they did not first die or undergo transplantation. . $2,23^{2}$

\section{Limitations}

This study was retrospective in nature and subject to the biases inherent in such analyses. In particular, this study was affected by selection bias caused by including only patients on whom BCPA had been performed, not all patients for whom BCPA was considered. This study was not designed or meant to describe interstage mortality or the rate of nonintervention for all patients who in the future might be candidates for BCPA for anatomic and physiologic reasons. Therefore, the results of this study cannot be extrapolated to all patients for whom BCPA would be considered in the future.

Echocardiographic data were limited to 117 patients since 1999, and thus data for 6 of 15 patients who died after BCPA are not available. Quantitative analyses of ventricular function, such as the measurement of $\Delta \mathrm{p} / \Delta \mathrm{t}$, were not performed at the time of the initial reading. ${ }^{24,25}$ Additionally, routine recordings of the angiographic grade of ventricular function or atrioventricular valve regurgitation were not available to validate echocardiographic findings.

Complete data for a greater number of patients, and in particular for those who died or underwent transplantation, would facilitate identification of other independent risk factors through multivariable modeling. The limited data in this study preclude the full evaluation of complex interactions that likely exist between the hemodynamic and morphologic variables in these patients.

\section{Conclusions}

Transplantation-free survival after BCPA is high when the procedure is used as a palliative before the Fontan procedure. At least moderate atrioventricular valve regurgitation is a significant risk factor for death or transplantation. The elucidation of the complex relationships between ventricular function and atrioventricular valve regurgitation and the exact mechanism of atrioventricular valve regurgitation in the patient with single-ventricle physiology are important goals of future investigations. Careful consideration is needed to determine the best long-term management of patients determined to be at high risk.

\section{References}

1. Alphonso N, Baghai M, Sundar P, Tulloh R, Austin C, Anderson D. Intermediate-term outcome following the Fontan operation: a survival, functional and risk-factor analysis. Eur J Cardiothorac Surg. 2005;28: 529-35.

2. Bridges ND, Jonas RA, Mayer JE, Flanagan MF, Keane JF, Castañeda AR. Bidirectional cavopulmonary anastomosis as interim palliation for high-risk Fontan candidates. Early results. Circulation. 1990; 82(5 Suppl):IV170-6.

3. Hawkins JA, Shaddy RE, Day RW, Sturtevant JE, Orsmond GS, McGough EC. Mid-term results after bidirectional cavopulmonary shunts. Ann Thorac Surg. 1993;56:833-7.

4. Pridjian AK, Mendelsohn AM, Lupinetti FM, Beekman RH 3rd, Dick M, Serwer G, et al. Usefulness of the bidirectional Glenn procedure as staged reconstruction for the functional single ventricle. Am J Cardiol. 1993;71:959-62.

5. Rubin DB. Multiple imputation for nonresponse in surveys. 1st ed. New York: John Wiley \& Sons; 1987.

6. Schafer JL. Analysis of incomplete multivariate data. 1st ed. London: Chapman \& Hall; 1997.

7. Van Buuren S, Boshuizen HC, Knook DL. Multiple imputation of missing blood pressure covariates in survival analysis. Stat Med. 1999; 18:681-94.

8. Concato J, Peduzzi P, Holford TR, Feinstein AR. Importance of events per independent variable in proportional hazards analysis I. Background, goals, and general strategy. J Clin Epidemiol. 1995;48: 1495-501.

9. Peduzzi P, Concato J, Feinstein AR, Holford TR. Importance of events per independent variable in proportional hazards analysis II. Accuracy and precision of regression estimates. J Clin Epidemiol. 1995;48:1503-10.

10. Lin DY, Wei LJ, Ying Z. Checking the Cox model with cumulative sums of martingale-based residuals. Biometrika. 1993;80:557-72.

11. Van Buuren S, Oudshoorn CGM. Multivariate imputation by chained equations: MICE v1.0 user's manual. Report PG/VG2/00.038, TNO Prevention and Health, Leiden.

12. Alejos JC, Williams RG, Jarmakani JM, Galindo AJ, Isabel-Jones JB, Drinkwater D, et al. Factors influencing survival in patients undergoing the bidirectional Glenn anastomosis. Am J Cardiol. 1995;75:1048-50.

13. Gentles TL, Mayer JE Jr, Gauvreau K, Newburger JW, Lock JE, Kupferschmid JP, et al. Fontan operation in five hundred consecutive patients: factors influencing early and late outcome. J Thorac Cardiovasc Surg. 1997;114:376-91.

14. Mahle WT, Cohen MS, Spray TL, Rychik J. Atrioventricular valve regurgitation in patients with single ventricle: impact of the bidirectional cavopulmonary anastomosis. Ann Thorac Surg. 2001;72:831-5.

15. Ashburn DA, McCrindle BW, Tchervenkov CI, Jacobs ML, Lofland GK, Bove EL, et al. Outcomes after the Norwood operation in neonates with critical aortic stenosis or aortic valve atresia. J Thorac Cardiovasc Surg. 2003;125:1070-82.

16. Day RW, Orsmond GS, Sturtevant JE, Hawkins JA, Doty DB, McGough EC. Early and intermediate results of the Fontan procedure at moderately high altitude. Ann Thorac Surg. 1994;57:170-6.

17. Seliem MA, Baffa JM, Vetter JM, Chen SL, Chin AJ, Norwood WI Jr. Changes in right ventricular geometry and heart rate early after hemiFontan procedure. Ann Thorac Surg. 1993;55:1508-12.

18. Forbes TJ, Gajarski R, Johnson GL, Reul GJ, Ott DA, Drescher K, et al. Influence of age on the effect of bidirectional cavopulmonary anastomosis on left ventricular volume, mass and ejection fraction. J Am Coll Cardiol. 1996;28:1301-7.

19. Jacobs ML, Rychik J, Rome JJ, Apostolopoulou S, Pizarro C, Murphy JD et al. Early reduction of the volume work of the single ventricle: the hemi-Fontan operation. Ann Thorac Surg. 1996;62:456-61. 
20. Jayakumar KA, Addonizio LJ, Kichuk-Chrisant MR, Galantowicz ME, Lamour JM, Quaegebeur JM, et al. Cardiac transplantation after the Fontan or Glenn procedure. J Am Coll Cardiol. 2004;44:2065-72.

21. Michielon G, Parisi F, Squitieri C, Carotti A, Gagliardi G, Pasquini L, et al. Orthotopic heart transplantation for congenital heart disease: an alternative for high-risk Fontan candidates? Circulation. 2003;108 Suppl 1: II140-9.

22. Yeh T Jr, Williams WG, McCrindle BW, Benson LN, Coles JG, Van Arsdell GS, et al. Equivalent survival following cavopulmonary shunt: with or without the Fontan procedure. Eur J Cardiothorac Surg. 1999;16:111-6.
23. Day RW, Etheridge SP, Veasy LG, Jenson CB, Hillman ND, Di Russo $\mathrm{GB}$, et al. Single ventricle palliation: greater risk of complications with the Fontan procedure than with the bidirectional Glenn procedure alone. Int J Cardiol. 2006;106:201-10.

24. Rhodes J, Udelson JE, Marx GR, Schmid CH, Konstam MA, Hijazi $\mathrm{ZM}$, et al. A new noninvasive method for the estimation of peak $\mathrm{dP} / \mathrm{dt}$. Circulation. 1993;88:2693-9.

25. Rhodes J, Fulton DR, Levine JC, Marx GR. Comparison between the mean $\mathrm{dP} / \mathrm{dt}$ during isovolumetric contraction and other echocardiographic indexes of ventricular systolic function. Echocardiography. 1997; 14:215-22.

\section{ON THE MOVE?}

Don't miss a single issue of the journal! To ensure prompt service when you change your address, please photocopy and complete the form below.

Please send your change of address notification at least six weeks before your move to ensure continued service. We regret we cannot guarantee replacement of issues missed due to late notification.

\section{JOURNAL TITLE:}

Fill in the title of the journal here.

\section{OLD ADDRESS:}

Affix the address label from a recent issue of the journal here.

\section{NEW ADDRESS:}

Clearly print your new address here.

Name

Address

City/State/ZIP
COPY AND MAIL THIS FORM TO:

Elsevier Inc.

Subscription Customer Service

6277 Sea Harbor Dr

Orlando, FL 32887
OR FAX TO:

407-363-9661

OR E-mail:

elspcs@elsevier.com
OR PHONE:

800-654-2452

Outside the U.S., call

407-345-4000 


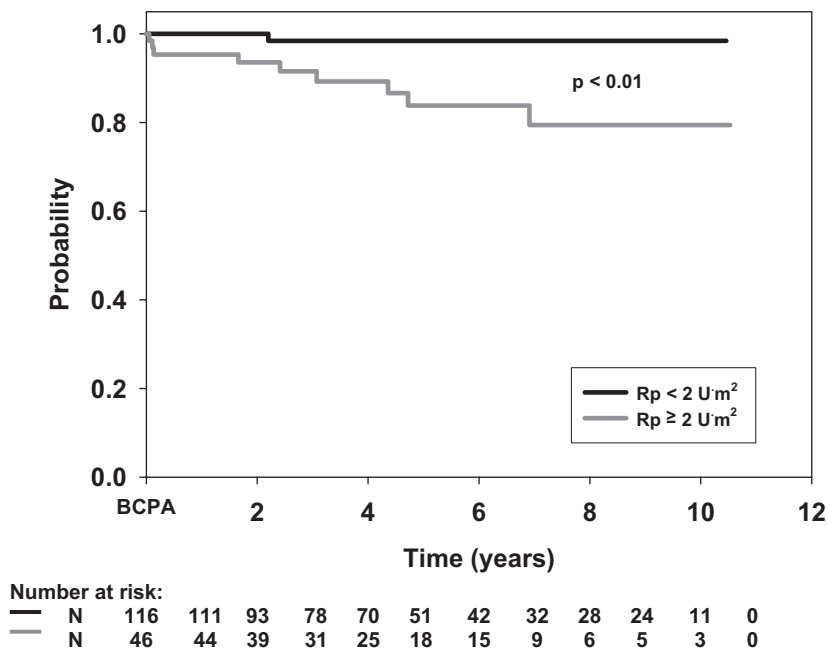

Figure E1. Kaplan-Meier plot of freedom from death or transplantation after bidirectional cavopulmonary anastomosis (BCPA) by classification of pulmonary vascular resistance $(R p)$.

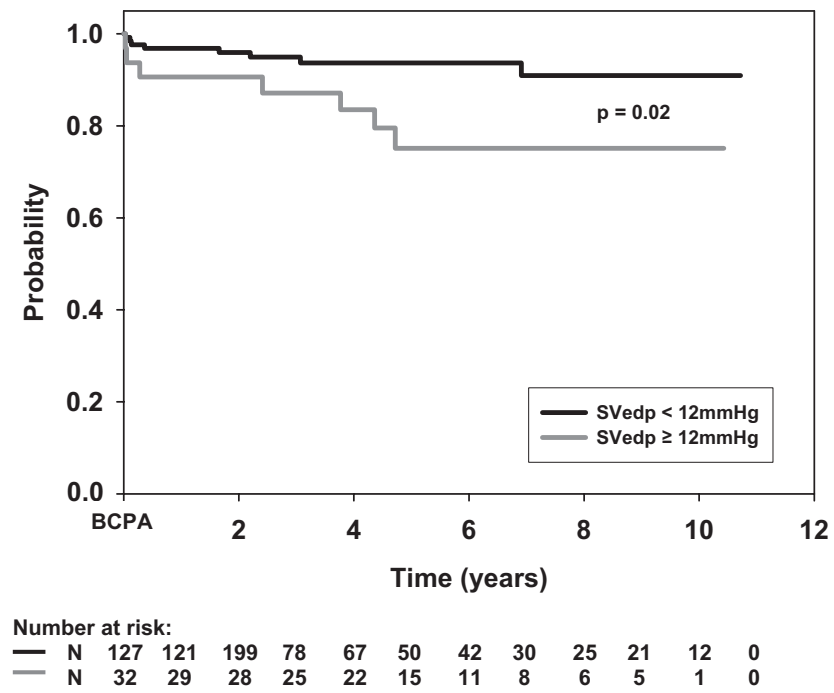

Figure E2. Kaplan-Meier plot of freedom from death or transplantation after bidirectional cavopulmonary anastomosis (BCPA) by classification of single-ventricle end-diastolic pressure (SVedp). 


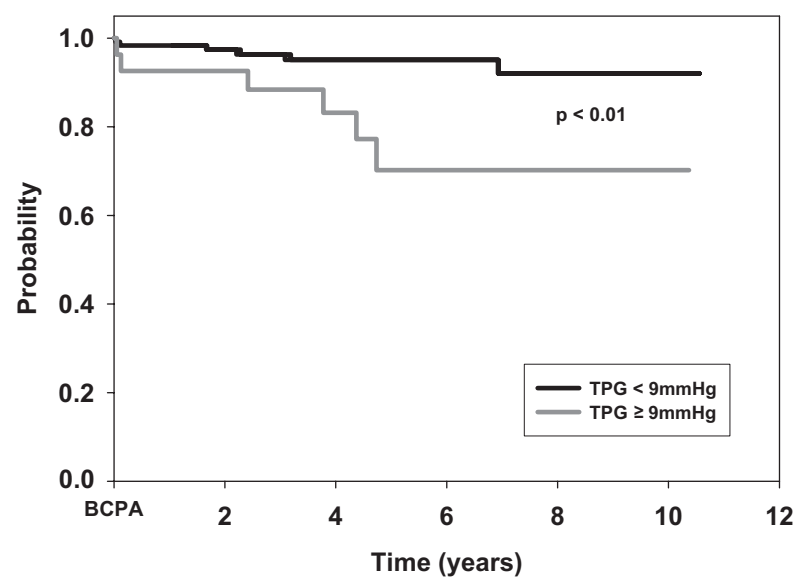

Number at risk:

\begin{tabular}{cccccccccccccc}
\multicolumn{2}{l}{ Number at risk: } & & & & & & & & & & \\
- & 118 & 113 & 90 & 73 & 63 & 45 & 35 & 24 & 22 & 18 & 8 & 0 \\
- & $N$ & 26 & 24 & 23 & 16 & 14 & 9 & 8 & 5 & 3 & 2 & 0 & 0
\end{tabular}

Figure E3. Kaplan-Meier plot of freedom from death or transplantation after bidirectional cavopulmonary anastomosis (BCPA) by classification of transpulmonary gradient (TPG).

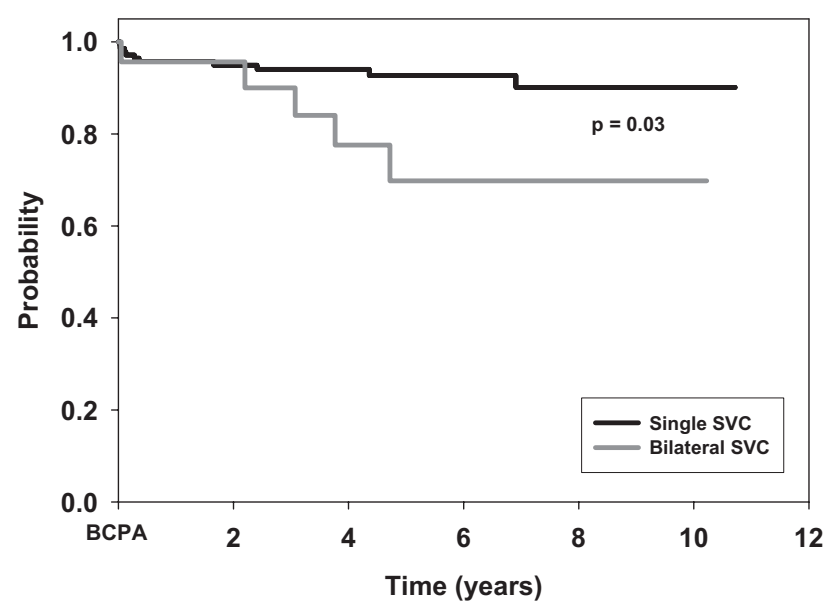

Number at risk:

$\begin{array}{ccccccccccccc}-\mathrm{N} & 144 & 133 & 112 & 92 & 81 & 59 & 46 & 32 & 27 & 24 & 12 & 0 \\ \mathrm{~N} & 23 & 22 & 19 & 15 & 12 & 9 & 9 & 7 & 5 & 3 & 1 & 0\end{array}$

Figure E4. Kaplan-Meier plot of freedom from death or transplantation after bidirectional cavopulmonary anastomosis (BCPA) by presence of presence of bilateral superior venae cavae (SVC).

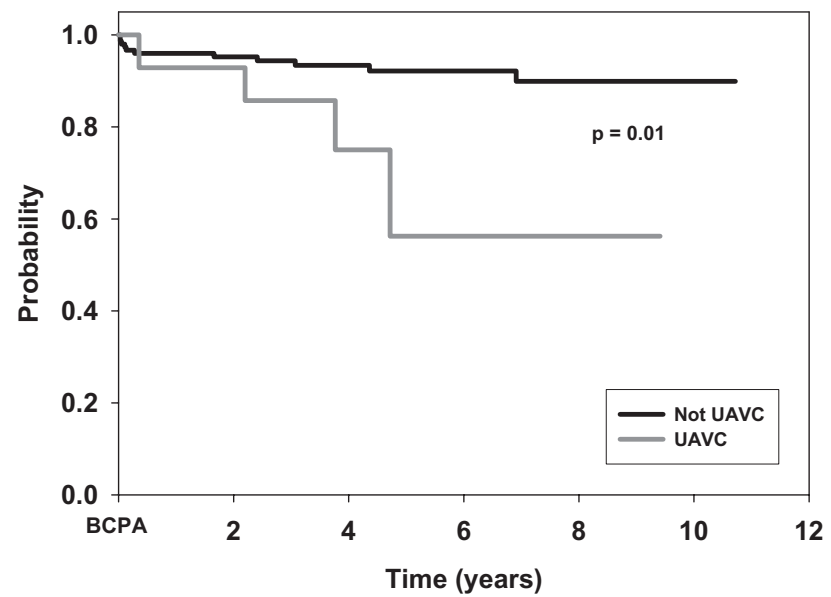

Number at risk:

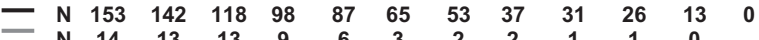

Figure E5. Kaplan-Meier plot of freedom from death or transplantation after bidirectional cavopulmonary anastomosis (BCPA) by presence of unbalanced atrioventricular canal (UAVC).

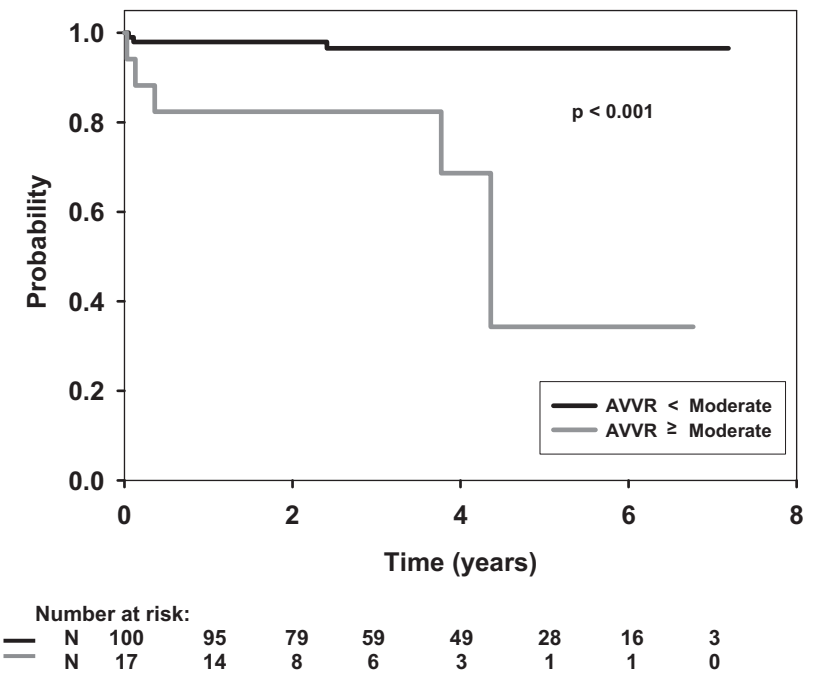

Figure E6. Kaplan-Meier plot of freedom from death or transplantation after bidirectional cavopulmonary anastomosis (BCPA) by degree of preoperative atrioventricular valve regurgitation (AVVR). 